\title{
LETTER
}

\section{Sr thermometer for Porites corals: Little need to measure Ca?}

\author{
Min Sun, ${ }^{1}$ CHIN-Hsin ChIU, ${ }^{2}$ CHUAN-CHOU Shen ${ }^{2,3 *}$ and TyPHOON LeE ${ }^{2}$ \\ ${ }^{1}$ Department of Earth Sciences, University of Hong Kong, Pokfulam Road, Hong Kong \\ ${ }^{2}$ Institute of Earth Sciences, Academia Sinica, Taipei, Taiwan \\ ${ }^{3}$ School of Oceanography, University of Washington, Seattle, WA 98195 , U.S.A.
}

(Received February 8, 1999; Accepted June 7, 1999)

\begin{abstract}
Cubic sub-samples of $1 \mathrm{~mm}^{3}$ (about $0.5 \mathrm{mg}$ ) were obtained from a Porites coral slice collected from Xisha Island, South China Sea, and analysed for Sr and Ca concentrations by ID-TIMS after precise weighing. Ca contents in the coral are quite uniform (mean $=38.16 \mathrm{wt} \%, 2 \sigma=0.11 \mathrm{wt} \%$ ). The variation corresponds to $\pm 0.4^{\circ} \mathrm{C}$, about the level of uncertainty of the $\mathrm{Sr} / \mathrm{Ca}$-SST calibration. Sr concentrations and $\mathrm{Sr} / \mathrm{Ca}$ ratios covary linearly, and the line can be defined by the equation $\mathrm{Sr} / \mathrm{Ca} \times 10^{3}=0.111 \mathrm{Sr}-0.5061$ (correlation coefficient $r=0.9955$ ), where $\mathrm{Sr}$ is in $\mu \mathrm{mol} / \mathrm{gm}$. Consequently, our results demonstrate that the previously established coral $\mathrm{Sr} / \mathrm{Ca}$ thermometer, $\mathrm{Sr} / \mathrm{Ca} \times 10^{3}=\mathrm{A}+\mathrm{B} \times \mathrm{T}$, can be simplified as a $\mathrm{Sr}$ thermometer, $0.111 \mathrm{Sr}-0.5061=\mathrm{A}+\mathrm{B} \times \mathrm{T}$, where $\mathrm{Sr}$ is in $\mu \mathrm{mol} / \mathrm{gm}$. Therefore, measurement of $\mathrm{Ca}$ in corals could be unnecessary. If different levels of impurity exist in different corals, a few Ca measurements in each coral may be needed, but definitely there is no need to measure every sub-sample.
\end{abstract}

\section{INTRODUCTION}

Trace elements in corals, the natural archives, have been widely used in reconstructing variations of environmental parameters and anthropogenic contaminations around coastal and pelagic zones in past tropical oceans (e.g., Shen and Boyle, 1987; Beck et al., 1992, 1997; Guilderson et al., 1994; Gagan et al., 1998). About two decades ago, Sr/ $\mathrm{Ca}$ ratios in coral aragonite skeletons were proven to be directly related to Sea Surface Temperature (SST). Smith et al. (1979) suggested that $\mathrm{Sr} / \mathrm{Ca}$ ratios in coral skeletons can be used as a proxy for SST. However, because of small seasonal variations (about $2-4 \%$ ) in coral $\mathrm{Sr} / \mathrm{Ca}$ ratios, precision of data was not satisfactory until recent years. A high precision $\mathrm{Sr} / \mathrm{Ca}$ coral thermometer was first established by Beck et al. (1992), and further calibrated by de Villiers et al. (1994), Shen et al. (1996a), Alibert and McCulloch (1997), and
Gagan et al. (1998). These recent studies employed isotope dilution-thermal ionization mass spectrometer (ID-TIMS) technique. This technique provides high quality data, so SST in the past can be reconstructed with an accuracy better than $0.5^{\circ} \mathrm{C}$. In addition, ID-TIMS requires only very small quantities of sub-samples for the measurements. Consequently, reconstruction of SST with high resolution (monthly or even weekly) is now possible. However, a vital obstacle exists for reconstruction of long time period SST with such high resolution (monthly or weekly), by using this method. Since too many sub-samples need to be analyzed for $\mathrm{Sr}$ and $\mathrm{Ca}$, it will be time consuming and very costly.

Calcium is a major constituent element in coral skeletons. Obviously if its variation in a coral sample is negligible at the level of ID-TIMS Sr analytical error, or Ca concentrations can be determined by a fast method, we can greatly speed up

*Present address: Department of Geology and Geophysics, University of Minnesota, MN 55455, U.S.A. 
studies in this important field. Unfortunately, $\mathrm{Ca}$ variations in corals have not yet been investigated, because previous ID-TIMS studies only provide precise $\mathrm{Sr} / \mathrm{Ca}$ ratios, but often not accurate $\mathrm{Sr}$ or $\mathrm{Ca}$ concentrations. The reason is that the $\mathrm{Sr} / \mathrm{Ca}$ thermometer does not require precise weighing each of the sub-sample, since weights will be cancelled in the calculation of $\mathrm{Sr} / \mathrm{Ca}$ ratios. Moreover, in order to achieve monthly or even weekly resolution, the quantity of each coral sub-sample is extremely small, which makes precise weighing difficult. So precise weighing is not common in coral $\mathrm{Sr} / \mathrm{Ca}$ studies.

In this study, with the help of a special subsampling technique and using a high precision micro-balance, we obtained accurate data for both $\mathrm{Sr} / \mathrm{Ca}$ ratios and concentrations of $\mathrm{Sr}$ and $\mathrm{Ca}$ in a Porites coral collected from Xisha Island, South China Sea. The results enable us to examine $\mathrm{Ca}$ variation in the coral sample, and we found that the $\mathrm{Sr} / \mathrm{Ca}$ thermometer can be simplified to a $\mathrm{Sr}$ thermometer.

\section{Methodology}

A specially-designed micro-surgical machine, CHINA-2 (Shen et al., 1996b), was used in this study. The sample slice was placed on a micrometer-controlled 3-D stage, a high speed fine circular diamond saw fixed over the stage can cut the sample in any direction. Cubic sub-samples of 1 $\mathrm{mm}^{3}$ (about $0.5 \mathrm{mg}$ ) were obtained along the maximum growth direction of the Porites coral slice from Xisha Island. This sampling technique allows us to clean each sub-sample after cutting, and makes precise weighing easier. A CAHN C-31 micro-balance was utilized for sample weighing (precision $0.1 \mu \mathrm{g}$ ).

Sub-samples were dissolved in $1 \mathrm{~N} \mathrm{HCl}$, and then small aliquots were mixed with ${ }^{42} \mathrm{Ca}-{ }^{44} \mathrm{Ca}$ ${ }^{84} \mathrm{Sr}$ triple spike. Concentrations of $\mathrm{Sr}$ and $\mathrm{Ca}$ were measured on a VG-354 mass spectrometer at the Institute of Earth Sciences, Academia Sinica, following the method described by Shen (1996) and Shen et al. (1996a).

\section{RESUlTS AND DISCUSSION}

High precision monthly coral Ca variation

Two hundred and one Porites coral sub-samples from Xisha were measured for $\mathrm{Sr}$ concentrations. They cover a time-interval of 18 years (1977-1994). After obtaining Sr data, 61 remaining aliquots of these sub-samples were carefully chosen for Ca determination so that at least those with maximum and minimum $\mathrm{Sr}$ concentrations for each year would also have Ca data. Fourteen continuous sub-samples show trivial seasonal $\mathrm{Ca}$ variations $(<0.22 \%)$ in the coral skeleton (Fig. 1). Our overall results also indicate that $\mathrm{Ca}$ contents in the coral are quite uniform (mean $=38.16 \mathrm{wt} \%$, $2 \sigma=0.11 \mathrm{wt} \%)$. The variation corresponds to

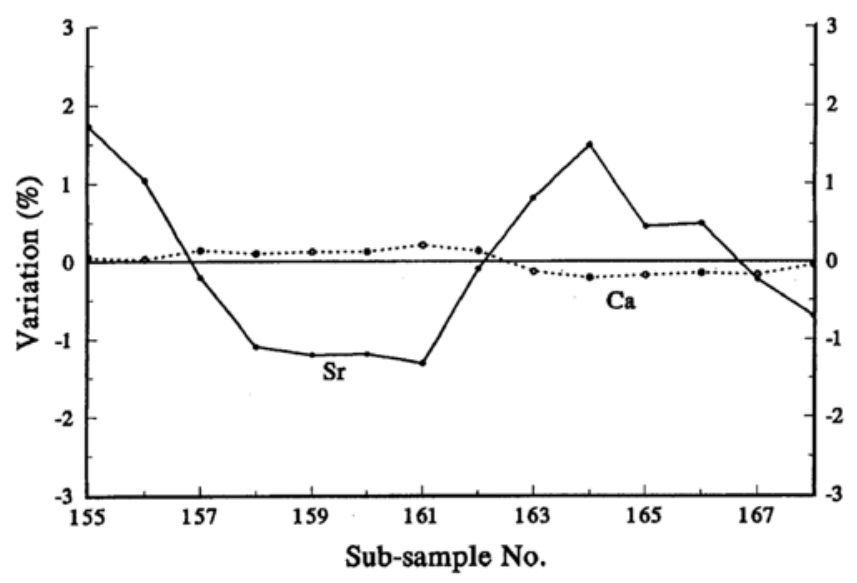

Fig. 1. Variation of Sr and Ca contents in 14 continuous sub-samples from Xisha Porites coral. 
$\pm 0.4^{\circ} \mathrm{C}$, about the level of uncertainty of the $\mathrm{Sr} /$ Ca-SST calibration (e.g., Shen et al., 1996a). Therefore, measurement of $\mathrm{Ca}$ in corals may not be necessary.

In order to investigate the consistency of variations of $\mathrm{Ca}$ in coral samples from different localities, the same laboratory also measured a modern Porites coral sampled from Lutao offshore, southeastern Taiwan and a Porites fossil coral from Shi-Zu Profile in Kenting, southern Taiwan. The modern coral from Lutao possesses Ca contents identical to that from Xisha $(38.18 \mathrm{wt} \%$, $2 \sigma=0.14 \mathrm{wt} \%$ ). However the $3050 \pm 70$ year fossil coral has higher Ca contents ( $38.47 \mathrm{wt} \%, 2 \sigma=$ $0.05 \mathrm{wt} \%$ ). This difference is either due to very small degree of recrystallization of the coral aragonite, which is difficult to detect by X-ray diffraction analysis, or can be attributed to different levels of impurities in different corals. If the former is the reason, the sample would not be suitable for SST study, no matter how the Ca varies; if the latter is true, some $\mathrm{Ca}$ measurements are required for each coral sample. This question can be addressed by future studies. Nevertheless, even a few $\mathrm{Ca}$ measurements in each coral may be needed, but definitely there is no need to measure every sub-sample.

\section{Sr thermometry}

Mainly because the Ca concentrations are uni- form, Sr data from the Xisha coral co-vary with $\mathrm{Sr} / \mathrm{Ca}$ ratios with a correlation coefficient $r=$ 0.9955 . The linear equation is $\mathrm{Sr} / \mathrm{Ca} \times 10^{3}=$ $0.111 \mathrm{Sr}-0.5061$, where $\mathrm{Sr}$ is in $\mu \mathrm{mol} / \mathrm{gm}$ (Fig. 2). Consequently, our results demonstrate that the previously established $\mathrm{Sr} / \mathrm{Ca}$ thermometer, $\mathrm{Sr} / \mathrm{Ca} \times 10^{3}=\mathrm{A}+\mathrm{B} \times \mathrm{T}$ (e.g., Beck et al., 1992), can be simplified to a $\mathrm{Sr}$ thermometer, $0.111 \mathrm{Sr}$ $-0.5061=\mathrm{A}+\mathrm{B} \times \mathrm{T}$, where $\mathrm{Sr}$ is in $\mu \mathrm{mol} / \mathrm{gm}$. Because of the trivial seasonal Ca variation in the coral skeleton (Fig. 1), this equation should be more precise than simply substituting the average $\mathrm{Ca}$ content into the $\mathrm{Sr} / \mathrm{Ca}$ thermometer.

Because ID-TIMS can provide precise analysis for small quantities of each sub-sample, past SST records can be reconstructed for monthly or even weekly resolution by coral $\mathrm{Sr} / \mathrm{Ca}$ proxy. However, this means thousands of sub-samples need to be analyzed for a hundred-year record, using this time-consuming and high-cost technique. Our study indicates that it is now possible to save many (if not all) instrumental analyses for $\mathrm{Ca}$ concentrations. Therefore the total number of analyses can be reduced up to $50 \%$. More significantly, Sr ID-TIMS analysis is routine work in numerous laboratories, whereas mainly because of the serious mass fractionation during mass spectrometer measurement, only a few isotope laboratories in the world can analyze $\mathrm{Ca}$. This has greatly hampered coral paleo-climate studies. If

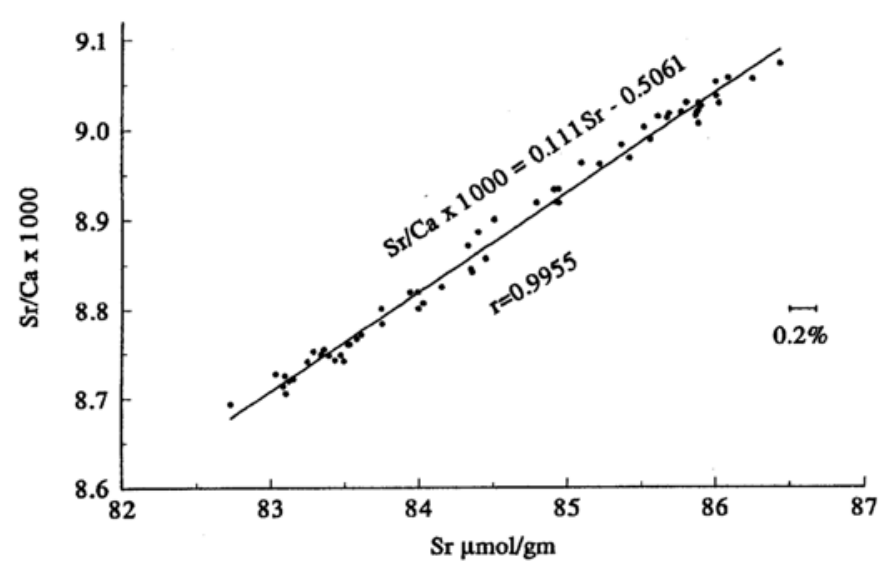

Fig. 2. Sr vs. Sr/Ca diagram for 61 subsamples from a Porites coral from Xisha Island. The Sr/Ca ratio of a subsample can be calculated from its $\mathrm{Sr}$ concentration within analytical error. 
it is possible to avoid most, or all, Ca analyses, many more studies can be carried out, and this will definitely speed up our understanding of global change in the past.

Furthermore, Ca measurements can also be saved in other trace element studies on corals. For example, seasonal variations of $\mathrm{Mn} / \mathrm{Ca}$ ratios in corals are about $30 \%$ (Shen et al., 1991), those of $\mathrm{Mg} / \mathrm{Ca}$ ratios are 25\% (Mitsuguchi et al., 1996), $\mathrm{U} / \mathrm{Ca}$ ratios vary $17 \%$ (Min et al., 1995), and Ba/ Ca ratios vary $15 \%$ (Lea et al., 1989). We suggest that these variations are also almost entirely caused by the intrinsic differences in concentrations of the trace elements. Therefore we should be able to keep the similar precision in the trace element to $\mathrm{Ca}$ ratio studies, but save time and cost if $\mathrm{Ca}$ measurement is omitted.

\section{CONCLUSIONS}

The Ca concentrations in the Porites coral from Xisha Island are nearly uniform, and the $\mathrm{Sr}$ concentrations covary linearly with the $\mathrm{Sr} / \mathrm{Ca}$ ratios. $\mathrm{The} \mathrm{Sr} / \mathrm{Ca}$ coral thermometer can be simplified to a Sr thermometer. Therefore Ca measurements are not always necessary in coral studies.

Acknowledgments-We are grateful for C. H. Chen in the Institute of Earth Sciences, Academia Sinica, Taipei, Taiwan, who kindly transferred the technology of the micro-surgical technique to our coral subsampling. The coral sample was collected by B. F. $\mathrm{Nie}$, South China Sea Institute of Oceanography, Chinese Academy of Science. Discussion with G. T. Shen and J. J. Shen greatly benefited this paper. We thank Rob Kerrich and an anonymous reviewer for their constructive comments on an earlier version of the manuscript. This study was substantially supported by a grant from the Research Grants Council of the Hong Kong Special Administrative Region, China (HKU510/96P) to MS and partly supported by a NSC grant to TL (NSC 89-2116-M-001-026).

\section{REFERENCES}

Alibert, C. and McCulloch, M. T. (1997) Strontium/ calcium ratios in modern Porites corals from the Great Barrier Reef as a proxy for sea surface temperature: calibration of the thermometer and monitoring of ENSO. Paleoceanography 12, 345-363.
Beck, J. W., Edwards, R. L., Ito, E., Taylor, F. W., Recy, J., Rougerie, F., Joannot, P. and Henin, C. (1992) Seasurface temperature from coral skeletal strontium/ calcium ratios. Science 257, 644-647.

Beck, J. W., Recy, J., Taylor, F., Edwards, R. L. and Cabioch, G. (1997) Abrupt changes in early Holocene tropical sea surface temperature derived from coral records. Nature 385, 705-707.

de Villiers, S., Shen, G. T. and Nelson, B. K. (1994) $\mathrm{Sr} / \mathrm{Ca}$ thermometry in corals: method calibration and evaluation of $(\mathrm{Sr} / \mathrm{Ca})$ seawater and interspecies: variability. Geochim. Cosmochim. Acta 58, 197-208.

Gagan, M. K., Ayliffe, L. K., Hopley, D., Cali, J. A., Mortimer, G. E., Chappell, J., McCulloch, M. T. and Head, M. J. (1998) Temperature and surface-ocean water balance of the mid-Holocene tropical western Pacific. Science 279, 1014-1018.

Guilderson, T. P., Fairbanks, R. G. and Rubenstone, J. L. (1994) Tropical temperature variations since 20,000 years ago: Modulating interhemispheric climate change. Science 263, 663-665.

Lea, D. W., Shen, G. T. and Boyle, E. A. (1989) Coralline barium records temporal variability in equatorial Pacific upwelling. Nature 340, 373-376.

Min, G. R., Edwards, R. L., Taylor, F. W., Recy, J., Gallup, C. D. and Beck, J. W. (1995) Annual cycles of U/Ca in coral skeletons and U/Ca thermometry. Geochim. Cosmochim. Acta 59, 2025-2042.

Mitsugushi, T., Matsumoto, E., Abe, O., Uchida, T. and Isdale, P. J. (1996) Mg/Ca thermometry in coral skeletons. Science 274, 961-963.

Shen, C. C. (1996) High precision analysis of $\mathrm{Sr} / \mathrm{Ca}$ ratio and its environmental application. Ph.D. Dissertation, Tsing Hwa University.

Shen, C. C., Lee, T., Chen, C. Y., Wang, C. H., Dai, C. F. and $\mathrm{Li}, \mathrm{L}$. A. (1996a) The calibration of $\mathrm{D}[\mathrm{Sr} / \mathrm{Ca}]$ versus sea surface temperature relationship for porites corals. Geochim. Cosmochim. Acta 60, 3849-3858.

Shen, C. C., Lee, L. A., Dai, C. F. and Lee, T. (1996b) Improved subsampling method for biogenic skeletons and detailed growth pattern of porites coral. Proc. 4th Taiwan Conference of Coral Reef Biology, 246255.

Shen, G. T. and Boyle, E. A. (1987) Lead in corals: Reconstruction of historical industrial fluxes to the surface ocean. Earth Planet. Sci. Lett. 82, 289-304.

Shen, G. T. and Dunbar, R. B. (1995) Environmental controls on uranium in reef corals. Geochim. Cosmochim. Acta 59, 2009-2024.

Shen, G. T., Campbell, T. M., Dunbar, R. B., Willington, G. M., Colgan, M. W. and Glynn, P. W. (1991) Paleochemistry of manganese in corals from the Galapagos Islands. Coral Reefs 10, 91-101.

Smith, S. V., Buddemeier, R. W., Redalje, R. C. and Houck, J. E. (1979) Strotium-calcium thermometry in coral skeletons. Science 204, 404-407. 